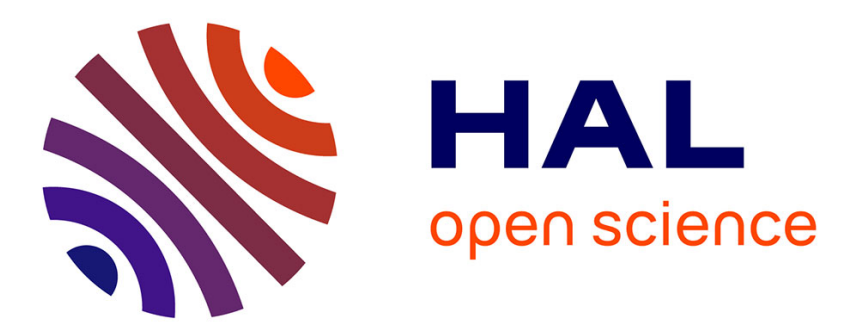

\title{
Dynamics effects on natural frequencies in modal analysis of PKMs
}

\author{
Julien Prades, Franck Jourdan, Olivier Company, Sébastien Krut, François \\ Pierrot
}

\section{> To cite this version:}

Julien Prades, Franck Jourdan, Olivier Company, Sébastien Krut, François Pierrot. Dynamics effects on natural frequencies in modal analysis of PKMs. MMAR: Methods and Models in Automation and Robotics, Aug 2015, Miedzyzdroje, Poland. pp.300-305, 10.1109/mmar.2015.7283891 . lirmm01275362

\section{HAL Id: lirmm-01275362 \\ https://hal-lirmm.ccsd.cnrs.fr/lirmm-01275362}

Submitted on 4 Apr 2016

HAL is a multi-disciplinary open access archive for the deposit and dissemination of scientific research documents, whether they are published or not. The documents may come from teaching and research institutions in France or abroad, or from public or private research centers.
L'archive ouverte pluridisciplinaire HAL, est destinée au dépôt et à la diffusion de documents scientifiques de niveau recherche, publiés ou non, émanant des établissements d'enseignement et de recherche français ou étrangers, des laboratoires publics ou privés. 


\title{
Dynamics Effects on Natural Frequencies in modal analysis of PKMs
}

\author{
Julien Prades*, Franck Jourdan ${ }^{\dagger}$, Olivier Company*, Sébastien Krut* and François Pierrot* \\ * Department of Robotics of LIRMM, University of Montpellier and CNRS \\ $\dagger$ LMGC, University of Montpellier and CNRS
}

\begin{abstract}
In this paper a new linearized dynamical model applied to PKM will be presented. This model allows us to do modal analysis by taking into account the dynamics of the robot. Finally, its influences on natural frequencies of robots will be highlighted on two numerical exemples.
\end{abstract}

\section{INTRODUCTION}

Parallel robots are said to be faster and stiffer than their serial counterparts. They benefit from continuous technical improvments and their mechanical performances are more impressive, as we can see in [1] (robot LIRMM $100 \mathrm{~g}$ ). Inertia effects due to speed and acceleration are more significant on the mecanism. Duringa path tracking sequency, positions, speeds and accelerations are calculated. Actuated torques can be deduced in respect with dynamical model. Then the command set up all the previous datas and the closed loop of control behave as "spring" in regards to actuations. Errors on following, speed and acceleration of the Mobile Platform will induce error on inertia forces and on the command. This paper introduce a methode to allow a quatification of the vibrations and will bring to light their effect on natural frequencies of the robots.

Inertia forces of the robot are linked with geometry weight and acceleration. During apick and place strategy, the goal is to move from a point $\mathrm{A}$ to a point $\mathrm{B}$ in the quickest way possible. This induces high inertia forces and leads the system to vibrate. In order to calculate the vibration frequencies, the mass and stiffness matrices must been known for the robot industriel. These matrices depend on the position. Andreas Müller [5] shown that the stiffness matrix can be modified by external forces, command forces and speed.

In this paper, the influence of acceleration on stiffness of the robots and therefore on their vibration frequencies will be explain.

This paper is organized as follows. In the next section, we describe the family of redundant parallel manipulators we are focusing on. Then, a simplified dynamic model based on jacobian matrices is derived. In section IV, the proposed analysis methodology is presented, based on the linearization of the dynamic model. The following section gives preliminary simulation results for two manipulators and provides some comments.

\section{PKMs Concerned With THE STUdy}

This study is focused on a specific type of robots, light ones. Moreover we are considering fast ones with parallel kinematic structure that are redundantly in actuation. As an example, we can consider the Par2 in [2]. The robot has, $u$ actuated joints and its Mobile Platform (MP) has $v$ degrees of freedom. The structure has also $u$ kinematic chains between its base frame and the MP with $u \geq v$ due tue the actuation redundancy. The objective of this paper is to determinate the impact of the dynamics on robot eigen frequencies for such parallel robots.

In this paper we are considering robots with the following architecture:

- Kinematic chains are composed by two solids and three joints

- Actuators can be linear motors or rotative motors, they are modelized by the $L_{1 i}$ joints which can be revolute or primatic

- $L_{2 i}$ et $L_{3 i}$ joints can be revolute in the case of a planar mechanism or spherical (or universal) for spatial mechanisms

The robot generic joint graph is shown in figure 7. According to this description, we can mention that this family of robots includes R4 Par2 and Dual-V see [1] and [2] and [3]

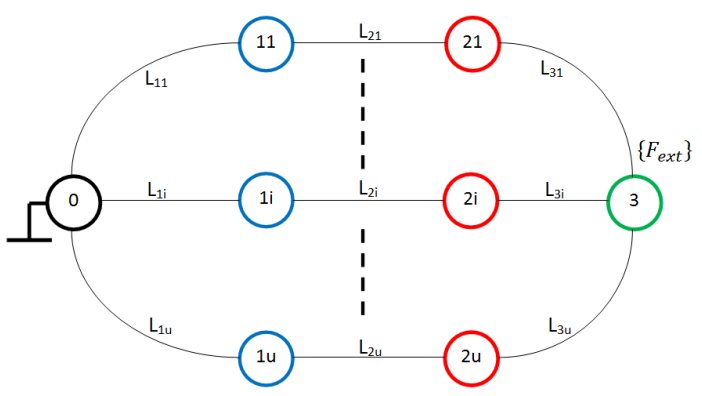

Fig. 1. Robots type generic joint graph

The bars mass and inertia are neglected compared to its motors and MP inertia. This structure mass repartition allows us to formulate the following simplifying hypothesis 1 :

The constant mass repartition of intermediate bars is replaced by two pinpoint masses weighting each half of the bars mass at their ends. Their mass and inertia are included in the adjacent parts mass. The paper [1] use also this hypothesis.

Hypothesis 1 allows to say that bars are only stressed with tension or compression actions. It follows that :

- Bars dynamics are neglected

- Actuators dynamics and MP study are simplified and can be done separately as we will see in section III of this paper

- The robot dynamics equation can be written using only the Jacobian matrix of the robot (the same as the one used in kinematic study) 
This is the key to have simple equations.

\section{PRELEMINARY SIMPLIFIED DYNAMIC MODEL}

Hypothesis 1 allows to write the Dynamic Model related to Jacobian Matrices. Adding the following hypothesis : frictions is neglected, solids keep their shapes and the links are perfect. Preliminary calculus will start with the writing of Inverse Geometric Model(IGM) before establishing robot Dynamic equations. Robot kinematic study gives IGM which links operational coordinates vector $\underline{X}$ of $\mathrm{MP}$ to actuated joint coordinates vector $q$.

$$
\underline{\underline{J_{q}}} \underline{\dot{q}}=\underline{\underline{J_{x}}} \underline{\dot{X}}
$$

As $J_{q}$ is a full rank square matrix (away from singular configurations), for our family of robots we can express:

$$
\underline{\dot{q}}=\underline{\underline{J_{m}}} \underline{\dot{X}}
$$

with:

$$
\underline{\underline{J_{m}}}=\underline{\underline{J_{q}}} \underline{\underline{J_{x}}}
$$

Actuator dynamic equation is obtained by isolating the actuator moving parts. It is written depending on actuators inertia and bars inertia contribution $\underline{m}$, forces applied by bars $\underline{F_{b}}$ and actuators torque $\underline{\tau}$.

$$
\underline{\tau}+\underline{\underline{J_{q}^{T}}} \underline{F_{b}}=\underline{\underline{m}} \underline{\ddot{q}}
$$

MP dynamic equation is achieved by isolating it. It is written depending on platform inertia and bars inertia contribution $\underline{\underline{M}}$, forces applied by bars $\underline{F_{b}}$ and external forces $\underline{F_{\text {ext }}}$ :

$$
\underline{F_{\text {ext }}}-\underline{\underline{J_{x}^{T}}} \underline{F_{b}}=\underline{\underline{M}} \underline{\ddot{X}}
$$

By derivating the IGM with respect to time (eq. 2), connection between the two dynamic equations 4 and 5 is obtained

$$
\underline{\ddot{q}}=\underline{\underline{J_{m}}} \underline{\ddot{X}}+\underline{\underline{J_{m}}} \underline{\dot{X}}
$$

Which give us the complete robot dynamic model.

$$
\underline{F_{\text {ext }}}=\left(\underline{\underline{M}}+\underline{\underline{J_{m}^{T}}} \underline{\underline{m}} \underline{\underline{J_{m}}}\right) \underline{\ddot{X}}+\underline{\underline{J_{m}^{T}}} \underline{\underline{m}} \underline{\underline{J_{m}}} \underline{\dot{X}}-\underline{\underline{J_{m}^{T}}} \underline{\tau}
$$

where:

- $\underline{J_{q}}, \underline{J_{x}}$ and $\underline{J_{m}}$ matrices are written based on the following $\overline{\overline{\text { variables }} \underline{\bar{X}} \text { and } q}$

- $\dot{J}_{m}$ matrix is written based on the following variables $\underline{X}$, $\underline{\overline{\dot{X}}}$ and $\underline{q}, \underline{\dot{q}}$

For a sake of clarity, dependence of associated variables won't be specified, except in case where numericals values will have to be calculated. This point will be important in section IV where matrices numerical evaluation and derivation are necessary. Then we will have to keep in mind that $\underline{q}=f(\underline{X})$ and $\underline{\dot{q}}=f(\underline{X}, \underline{\dot{X}})$.

\section{Proposed Analysis Methodology}

Robot dynamic equation is non linear. To extract informations of phenomenons which interest us, meaning actuating and moving effects over natural frequencies, we apply first order Taylor formula near to functional point :

$$
P_{f}=\left(\underline{X_{0}}, \underline{\dot{X}_{0}}, \underline{\ddot{X}_{0}}, \underline{\tau_{0}}, \underline{F_{0}}\right)
$$

It should be noted that in this study we take into account torques in the functional point, as cable robot studies. To simplify the writing, we use function $h$ as :

$$
h=\left(\underline{\underline{M}}+\underline{\underline{J_{m}^{T}}} \underline{\underline{m}} \underline{\underline{J_{m}}}\right) \underline{\ddot{X}}+\underline{\underline{J_{m}^{T}}} \underline{\underline{m}} \underline{\underline{j_{m}}} \underline{\dot{X}}-\underline{\underline{J_{m}^{T}}} \underline{\tau}-\underline{F_{\text {ext }}}
$$

Taylor equation of $h$ gives :

$\left.\frac{\partial h}{\partial \underline{\ddot{X}}}\right|_{P_{f}} \Delta \underline{\ddot{X}}+\left.\frac{\partial h}{\partial \underline{\dot{X}}}\right|_{P_{f}} \Delta \underline{\dot{X}}+\left.\frac{\partial h}{\partial \underline{X}}\right|_{P_{f}} \Delta \underline{X}+\left.\frac{\partial h}{\partial \underline{\tau}}\right|_{P_{f}} \Delta \underline{\tau}+\left.\frac{\partial h}{\partial \underline{F_{e x t}}}\right|_{P_{f}} \Delta \underline{F_{e x t}}=0$

To go any further, we need informations about $\underline{\tau}$, which we can get thanks to the control law. In general, the simplest axis control is a proportional derivative controler (PDC). The PDC will be set in actuated joint coordinates to avoid singularity problems which can occur. PDC will be integrated in the equation. PDC law depends on joint position error $\underline{\varepsilon}$, joint speed error $\underline{\dot{\varepsilon}}$ and torques that don't cause movements of the MP. These torques allow to solve many problems like mechanical clearance and other issues [4]. Control law equation is expressed:

$$
\underline{\tau}(\dot{\varepsilon}, \varepsilon)=\underline{\tau_{0}}-\underline{\underline{K_{D}}} \underline{\underline{\varepsilon}}-\underline{\underline{K_{P}}} \underline{\underline{\varepsilon}}
$$

By studying the variations and supposing that control law stay constant near to the functional point $P_{f}$, we have :

$$
\Delta \underline{\tau}=-\underline{\underline{K_{D}}} \Delta \underline{\dot{q}}-\underline{\underline{K_{P}}} \Delta \underline{q}
$$

Using kinematic equations, we can write equation 13 depending on $\underline{X}$ :

$$
\Delta \underline{\tau}=-\underline{\underline{K_{D}}} \underline{\underline{J_{m}}} \underline{\underline{\dot{X}}}-\underline{\underline{K_{P}}} \underline{\underline{J_{m}}} \underline{\underline{X}}
$$

Looking at the equation terms, we notice that we get a second order differential equations system in $\Delta \underline{X}$ with constant coefficients depending on $P_{f}$. To simplify the writing, we will use matrices $\underline{\mathbb{M}}, \underline{\mathbb{C}}, \underline{\mathbb{K}}$ depending on inertia, Jacobian matrix and control law.

Then, we get the following equation:

$$
\Delta \underline{F_{\text {ext }}}=\underline{\underline{\mathbb{M}}} \Delta \underline{\ddot{X}}+\underline{\underline{\mathbb{C}}} \Delta \underline{\dot{X}}+\underline{\underline{\mathbb{K}}} \Delta \underline{X}
$$

Matrices $\underline{\underline{M}}, \underline{\mathbb{C}}$ and $\underline{\underline{K}}$ are now expressed:

$$
\left.\underline{\underline{\mathbb{M}}}\left(\underline{X_{0}}\right)=\underline{\underline{M}}+\underline{\underline{J_{m}^{T}}} \underline{\underline{X_{0}}}\right) \underline{\underline{m}} \underline{\underline{J_{m}}}\left(\underline{X_{0}}\right)
$$

Matrices $\underline{\mathbb{C}}$ and $\underline{\underline{K}}$ which are too complex are decomposed as follows: 
- $\mathbb{C}_{v}$, kinematic damping matrix depending on speed and $\overline{\overline{\text { position. }}}$

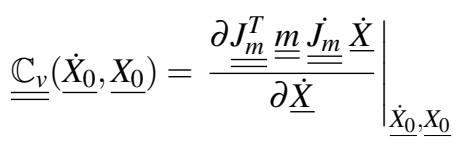

- $\mathbb{C}_{d}$, command law damping matrix depending on derivat$\overline{\overline{\text { ing }}}$ command law and position.

$$
\left.\underline{\underline{\mathbb{C}_{d}}}\left(\underline{X_{0}}\right)=\underline{\underline{J_{m}^{T}}} \underline{\underline{X_{0}}} \underline{\underline{K_{d}}} \underline{\underline{J_{m}}} \underline{\underline{X_{0}}}\right)
$$

- $\mathbb{K}_{i}$, dynamic stiffness matrix depending on acceleration $\overline{\overline{\text { and }}}$ position.

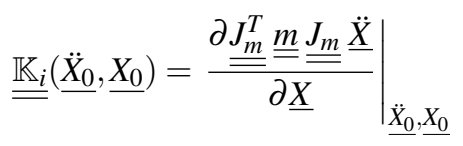

- $\underline{\underline{\mathbb{K}_{v}}}$, kinematic stiffness matrix depending on speed and $\overline{\text { position. }}$

$$
\underline{\underline{\underline{\mathbb{K}_{v}}}}\left(\underline{\dot{X}_{0}}, \underline{X_{0}}\right)=\left.\frac{\partial \underline{\underline{J_{m}^{T}}}}{\partial \underline{\underline{m}}} \underline{\underline{\underline{J_{m}}}} \underline{\underline{\dot{X}}}\right|_{\underline{\ddot{X}_{0}}, \underline{X_{0}}}
$$

- $\mathbb{K}_{g}$, geometrical stiffness matrix depending on torques $\overline{\overline{\text { and }}}$ position.

$$
\underline{\underline{\mathbb{K}_{g}}}\left(\underline{X_{0}}, \underline{\tau_{0}}\right)=-\left.\frac{\partial \underline{\underline{J_{m}^{T}}} \underline{\tau}}{\partial \underline{\underline{X}}}\right|_{\underline{X_{0}}, \underline{\tau_{0}}}
$$

- $\mathbb{K}_{a}$, proportional command stiffness matrix depending on

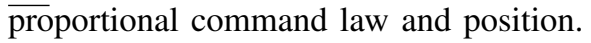

$$
\left.\underline{\underline{\mathbb{K}_{a}}}\left(\underline{X_{0}}\right)=\underline{\underline{J_{m}^{T}}} \underline{\underline{X_{0}}} \underline{\underline{K_{q}}} \underline{\underline{J_{m}}} \underline{\underline{X_{0}}}\right)
$$

$\mathbb{K}_{g}$ and $\mathbb{K}_{a}$ are the same matrices that in paper [6].

The different partial derivatives have to be done, taking into account that $q=f(\underline{X})$. We have therefore, for each partial derivatives of $\underline{w}$ :

$$
\left.\frac{\partial \underline{w}(\underline{X}, \underline{q}(\underline{X}))}{\partial \underline{X}}\right|_{P_{f}}=\left.\frac{\partial \underline{w}\left(\underline{X}, \underline{q_{0}}\right)}{\partial \underline{X}}\right|_{P_{f}}+\left.\frac{\partial \underline{w}(\underline{X}, \underline{q})}{\partial \underline{q}}\right|_{P_{f}} \underline{J_{m}}
$$

The equation 14 becomes :

$$
\Delta \underline{F_{\text {ext }}}=\underline{\underline{\mathbb{M}}} \Delta \underline{\ddot{X}}+\left(\underline{\underline{\mathbb{C}_{v}}}+\underline{\underline{\mathbb{C}_{d}}}\right) \Delta \underline{\dot{X}}+\left(\underline{\underline{\mathbb{K}_{i}}}+\underline{\underline{\mathbb{K}_{v}}}+\underline{\underline{\mathbb{K}_{g}}}+\underline{\underline{\mathbb{K}_{a}}}\right) \Delta \underline{X}
$$

These mathematical developments show the impact of the functional point $P_{f}$ over the different exposed matrices. These equations are able to describe the instant vibration behaviour of robot when it follows a trajectory, typically when there is a shock.

\section{VAlidation CASE Study}

\section{A. Type Analysis}

In this section, only numerical simulations will be presented. Two cases of robots natural frequencies are shown:

- $1^{\text {st }}$ case : for a given trajectory, during each interval, position and torques static functional point lowest natural frequency is calculated in static case.

- $2^{\text {nd }}$ case : for a given trajectory, during each interval, position, speed, acceleration and torques dynamic functional point lowest natural frequency is calculated in dynamic case.

We chose to work with redundant PKM easy to handle, but with Jacobian matrix which depends on the position. All studied robots structures can be modelized in a plane: PRR-4 and Dual-V manipulators, are both three degree of freedom robots $(v=3)$. MP motions are the two translations of the $(\vec{x}, \vec{y})$ plane plus the rotation about $\vec{z}$. Both manipulators have four actuation degrees $(u=4)$ :

- four translations for PRR-4

- four rotations for Dual-V

PRR-4 has been chosen and modelized to observe the impact of prestress and movement around one parallel singularity. Dual- $\mathrm{V}$ has been chosen because future experiments will be possible on an existing demonstrator. More details about these robots will be given in their respective subsection. To perform the calculations, the following trajectory has been chosen:

a translation along the axis $(O, \vec{y})$ beginning from $y_{d}=0.1 \mathrm{~m}$ to $y_{f}=-0.1 \mathrm{~m}$ by blocking $\vec{x}$ translation and $\vec{z}$ rotation.

Figures 2,3 and 4 show position, speed and acceleration with respect to time according to Table I.

\begin{tabular}{|c|c|}
\hline parametres & Values \\
\hline Jerk $_{\max }$ & $20000 \mathrm{~m} / \mathrm{s}^{3}$ \\
\hline Acceleration $_{\max }$ & $200 \mathrm{~m} / \mathrm{s}^{2}$ \\
\hline Vitesse $_{\max }$ & $10 \mathrm{~m} / \mathrm{s}$ \\
\hline Distance $_{\max }$ & $0.2 \mathrm{~m}$ \\
\hline
\end{tabular}

TABLE I

TRAJECTORY PARAMETERS

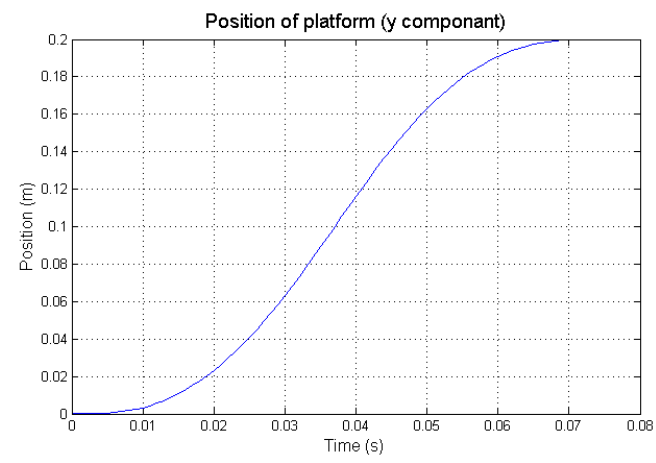

Fig. 2. Position over time 


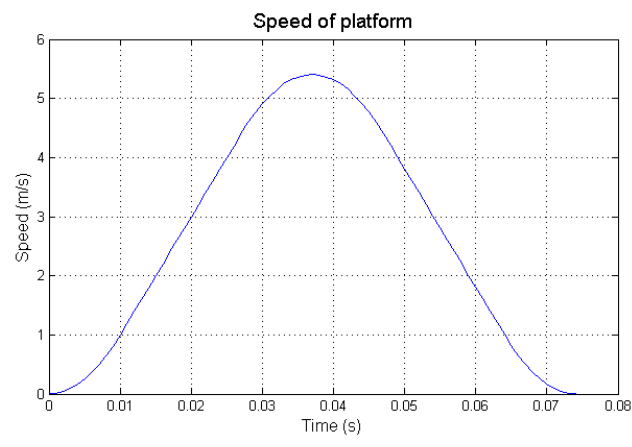

Fig. 3. Speed over time

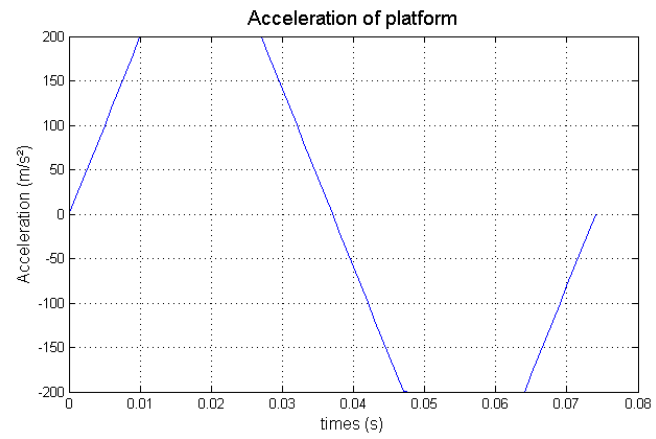

Fig. 4. Acceleration over time

To find natural frequencies, equation 23 terms have to be modified by cancelling $\Delta F_{e x t}$, which gives modal analysis typical equation. We can simplify the study by only calculating the natural frequency $f_{0}$ instead of calculating maximum amplitude vibration $f_{r}$, because both are close. This allows to cancel $\Delta \underline{\dot{X}}$ terms in equation 23 and becomes equation 24 .

$$
\underline{\underline{M}} \Delta \underline{\ddot{X}}+\left(\underline{\underline{\mathbb{K}_{i}}}+\underline{\underline{\mathbb{K}_{v}}}+\underline{\underline{\mathbb{K}_{g}}}+\underline{\underline{\mathbb{K}_{a}}}\right) \Delta \underline{X}=0
$$

Natural frequencies are obtained by finding eigenvalues of the following matrix.

$-1^{\text {st }}$ case $:$ static case

$$
\underline{\underline{M}}^{-1}\left(\underline{\underline{\mathbb{K}_{g}}}+\underline{\underline{\mathbb{K}_{a}}}\right)
$$

$\mathbb{K}_{g}$ is calculated with static torques only with redundant $\overline{\overline{\mathrm{PK}}} \mathrm{M}$ if not $\underline{\underline{\mathbb{K}_{g}}}=0$

$-2^{\text {nd }}$ case : dynamic case.

$$
\underline{\underline{M}}^{-1}\left(\underline{\underline{\mathbb{K}_{i}}}+\underline{\underline{\mathbb{K}_{v}}}+\underline{\underline{\mathbb{K}_{g}}}+\underline{\underline{\mathbb{K}_{a}}}\right)
$$

$\mathbb{K}_{g}$ is calculated with dynamic torques (redundant PKM $\overline{\overline{\text { could }}}$ have $\underline{\underline{J_{m}^{T}}}$ null space torques)

The only thing still needed is to formulate each robot matrices.

\section{B. Dual-V Case}

In this section the Dual-V case will be studied and robot design is shown in figure V-B. Actuation degrees are motors rotation at $A_{i}$ and operational coordinates vector is movements and rotation at $P_{5}$. For more details about Dual-V see reference [3]. We use the same Jacobian matrices. Geometry, inertia and control parameters are shown in table II.

\begin{tabular}{|c|c|c|c|}
\hline Parameters & Values & Paramètres & Values \\
\hline $\mathrm{a}$ & $0.39 \mathrm{~m}$ & $\mathrm{~m}$ & $0.0738 \mathrm{~kg} . \mathrm{m}^{2}$ \\
\hline $\mathrm{b}$ & $0.11 \mathrm{~m}$ & $\mathrm{M}$ & $2.1 \mathrm{~kg}$ \\
\hline $\mathrm{c}$ & $0.11 \mathrm{~m}$ & $\mathrm{I}$ & $0.0228 \mathrm{~kg} . \mathrm{m}^{2}$ \\
\hline$l_{i}$ & $0.28 \mathrm{~m}$ & $\mathrm{~K}$ & $900 \mathrm{~N} / \mathrm{rad}$ \\
\hline
\end{tabular}

TABLE II

GEOMETRIC, INERTIA AND COMMAND PARAMETERS OF DUAL-V

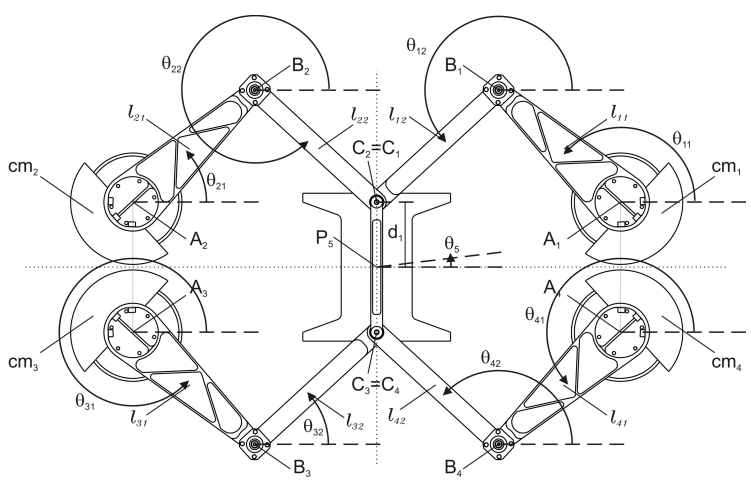

Fig. 5. Dual-V PKM

Kinematic study give the following matrices:

$$
\begin{gathered}
\underline{J_{q}}=-l\left(\begin{array}{cccc}
s\left(\theta_{11}-\theta_{12}\right) & 0 & 0 & 0 \\
0 & s\left(\theta_{21}-\theta_{22}\right) & 0 & 0 \\
0 & 0 & s\left(\theta_{31}-\theta_{32}\right) & 0 \\
0 & 0 & 0 & s\left(\theta_{41}-\theta_{42}\right)
\end{array}\right) \\
\underline{\underline{J_{x}}}=\left(\begin{array}{ccc}
c\left(\theta_{12}\right) & s\left(\theta_{12}\right) & -c\left(\theta_{12}-\theta_{5}\right) b \\
c\left(\theta_{22}\right) & s\left(\theta_{22}\right) & -c\left(\theta_{22}-\theta_{5}\right) b \\
c\left(\theta_{32}\right) & s\left(\theta_{32}\right) & c\left(\theta_{32}-\theta_{5}\right) b \\
c\left(\theta_{42}\right) & s\left(\theta_{42}\right) & c\left(\theta_{42}-\theta_{5}\right) b
\end{array}\right)
\end{gathered}
$$

The actuated coordinates vector is :

$$
\underline{q}=\left(\begin{array}{c}
\theta_{11} \\
\theta_{21} \\
\theta_{31} \\
\theta_{41}
\end{array}\right)
$$

The operational coordinates vector is :

$$
\underline{X}=\left(\begin{array}{l}
x_{5} \\
y_{5} \\
\theta_{5}
\end{array}\right)
$$

In Figure 6, first natural mode frequencies of Dual-V will be calculated every millisecond. The blue curve shows static case frequencies and the green curve shows dynamic case 
frequencies. When comparing both curves, we can see that acceleration decreases the frequency and that speed inscreases the frequency. Informations given by this model could help to control robots because it allows to evaluate moving robot natural frequency.

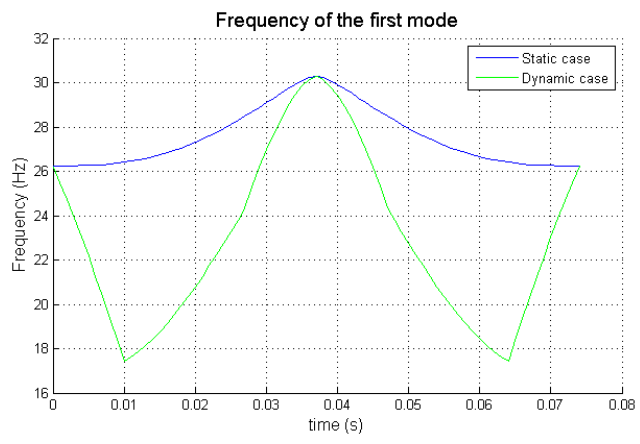

Fig. 6. First mode natural frequency of Dual-V

\section{PRR-4 Case}

Robot dimensions have been chosen in order to have a parallel singularity in the center of the workspace to see the wanted effect whatever working conditions are. Actuated joints are the prismatic ones with the $\vec{x}$ direction at point $A_{i}$. This actuation is done by linear motors (see Figure ). The robot kinematic scheme is given on Figure 7. Geometric, inertia and command parameters are shown in Table III.

\begin{tabular}{|c|c|c|c|}
\hline Parameters & Values & Parameters & Values \\
\hline $\mathrm{a}$ & $0.1 \mathrm{~m}$ & $\mathrm{~m}$ & $8 \mathrm{~kg}$ \\
\hline $\mathrm{b}$ & $0.1 \mathrm{~m}$ & $\mathrm{M}$ & $10 \mathrm{~kg}$ \\
\hline $\mathrm{e}$ & $1 \mathrm{~m}$ & $\mathrm{I}$ & $0.36 \mathrm{~kg} \cdot \mathrm{m}^{2}$ \\
\hline $\mathrm{l}$ & $1.27 \mathrm{~m}$ & $\mathrm{~K}$ & $8.10^{7} \mathrm{~N} / \mathrm{m}$ \\
\hline
\end{tabular}

TABLE III

GEOMETRIC, INERTIA AND COMMAND PARAMETERS OF PRR-4

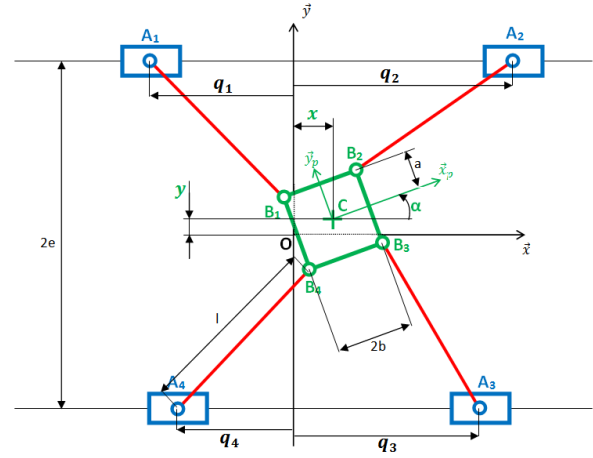

Fig. 7. PRR-4 robot parameters

$$
\underline{q}=\left(\begin{array}{l}
q_{1} \\
q_{2} \\
q_{3} \\
q_{4}
\end{array}\right)
$$

The operational coordinates vector is :

$$
\underline{X}=\left(\begin{array}{l}
x \\
y \\
\alpha
\end{array}\right)
$$

Robot Jacobian matrix will be found, based on bars speed (1 to 4 ) by using the following property for a rigid body (points $A$ and $B$ are supposed to belong to this body):

$$
\frac{d \overrightarrow{A B}}{d t} \cdot \overrightarrow{A B}=0
$$

Here, we chose $\vec{u}_{i}$ for bar i director vector from $A_{i}$ to $B_{i}$.

$$
\begin{gathered}
\underline{\underline{J_{q}}}=\left(\begin{array}{cccc}
\overrightarrow{u_{1}} \cdot \overrightarrow{x^{2}} & 0 & 0 & 0 \\
0 & \overrightarrow{u_{2}} \cdot \vec{x} & 0 & 0 \\
0 & 0 & \overrightarrow{u_{3}} \cdot \vec{x} & 0 \\
0 & 0 & 0 & \overrightarrow{u_{4}} \cdot \vec{x}
\end{array}\right) \\
\underline{\underline{J_{x}}}=\left(\begin{array}{ll}
{\overrightarrow{u_{1}}}^{T} & \left(\overrightarrow{u_{1}} \wedge \overrightarrow{B_{1} C}\right) \cdot \vec{z} \\
{\overrightarrow{u_{2}}}^{T} & \left(\overrightarrow{u_{2}} \wedge \overrightarrow{B_{2} C}\right) \cdot \vec{z} \\
{\overrightarrow{u_{3}}}^{T} & \left(\overrightarrow{u_{3}} \wedge \overrightarrow{B_{3} C}\right) \cdot \vec{z} \\
{\overrightarrow{u_{4}}}^{T} & \left(\overrightarrow{u_{4}} \wedge \overrightarrow{B_{4} C}\right) \cdot \vec{z}
\end{array}\right)
\end{gathered}
$$

In Figure 8, first natural mode frequencies of PRR-4 will be calculated every millisecond. The blue curve shows static case frequencies and the green curve shows dynamic case frequencies. When comparing both curves, we see the same kind of frequency behaviour. We also note that in static case, first mode frequency become null when $y=0$ (parallel singularity) but it does not nullify in the dynamic case. This method shows how we could go through singularities of this kind.

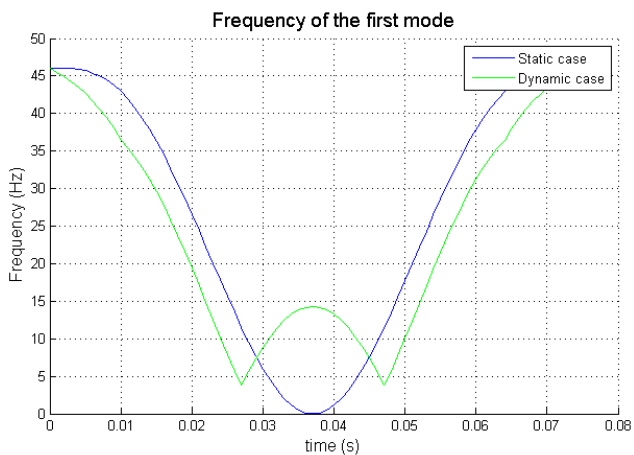

Fig. 8. First mode natural frequency of PRR-4

The actuated coordinates vector is : 


\section{CONCLUSION AND FUTUR WORK}

In this paper, we only have mathematical and numerical developments which show the impact of movements on robots natural frequency. Acceleration is harmful for first mode frenquency and speed has positive effect. The experimental confirmation of this model is in process with the Dual- $\mathrm{V}$ robot and another paper will show results of experiment. The next stage would be the improvement of the robots command by using informations given by this model.

\section{REFERENCES}

[1] Corbel D., Gouttefarde M., Company O., and Pierrot F., STowards 100G with PKM. Is actuation redundancy a good solution for pick-and-place?Ť 2010 IEEE International Conference on Robotics and Automation, Anchorage, Alaska, USA, May 3-8, 2010.

[2] Cédric Baradat, Vincent Nabat, Olivier Company, Sébastien Krut, François Pierrot. Par2: a Spatial Mechanism for Fast Planar, 2-dof, Pick-and-Place Applications. Fundamental Issues and Future Research Directions for Parallel Mechanisms and Manipulators, Sep 2009, Montpellier, France. pp.10, 2008.

[3] Volkert Van Der Wijk, Sébastien Krut, François Pierrot, Just Herder. Design and experimental evaluation of a dynamically balanced redundant planar 4-RRR parallel manipulator. The International Journal of Robotics Research, SAGE Publications (UK and US), 2013, pp.743758. <lirmm-00822698>

[4] A. Müller: Internal Preload Control of redundantly actuated Parallel Manipulators Ú Backlash avoiding Control, ICRA 2005

[5] A. Müller, ŞStiffness control of redundantly actuated parallel manipulators, $\breve{T}$ in Proc. IEEE Int. Conf. Robot. Autom., Orlando, FL, May 15Ǘ19, 2006, pp. 1153Ü1158

[6] S. Kock, W. Schumacher: A parallel $x-y$ manipulator with actuation redundancy for high-speed and active-stiffness applications, IEEE ICRA, Leuven, 1998, pp. 2295-2300 